\title{
EGF upregulates RFPL3 and hTERT via the MEK signaling pathway in non-small cell lung cancer cells
}

\author{
CHUAN LIN $^{1,2^{*}}$, YU QIN $^{3 *}$, HUA ZHANG $^{3}$, MING-YANG GAO $^{4}$ and YAN-FU WANG ${ }^{3}$ \\ ${ }^{1}$ Department of Respiratory Medicine, The First Affiliated Hospital of Dalian Medical University, Dalian, Liaoning 116000; \\ ${ }^{2}$ Department of Infectious Diseases, Jinhua Municipal Central Hospital, Jinhua, Zhejiang 321000; Departments of ${ }^{3}$ Geriatrics \\ and ${ }^{4}$ Dermatology, The First Affiliated Hospital of Dalian Medical University, Dalian, Liaoning 116000, P.R. China
}

Received August 23, 2017; Accepted March 23, 2018

DOI: $10.3892 /$ or.2018.6417

\begin{abstract}
Activation of the epidermal growth factor receptor (EGFR) during tumor development can trigger the MEK signaling pathway. In the present study, we investigated the MEK signaling pathway in non-small cell lung cancer (NSCLC) cells with respect to the effect of epidermal growth factor (EGF) on expression of Ret finger protein like 3 (RFPL3) and human telomerase reverse transcriptase (hTERT), and the effect of RFPL3 overexpression on other MEK signaling proteins. In vitro, A549 and H1299 cells were treated with different concentrations of EGF for $24 \mathrm{~h}$ or $48 \mathrm{~h}$. Expression of RFPL3 and hTERT at the mRNA and protein levels was determined by real-time quantitative PCR (RT-qPCR) and western blot analysis; cell viability was detected by MTT assay, and apoptosis was assayed via flow cytometry. We also pretreated A549 and H1299 cells with EGFR tyrosine kinase inhibitors, AG1478 and erlotinib, and MEK-specific inhibitor (PD98059) in the presence of EGF. We used western blot analysis to assess the expression levels of RFPL3, hTERT and related MEK-pathway proteins in A549 and H1299 cells transfected with RFPL3-overexpression plasmids. EGF significantly upregulated RFPL3 and hTERT protein levels in the NSCLC cells. RFPL3 and hTERT proteins upregulation by EGF were attenuated by pretreatment with AG1478 and erlotinib. EGF promoted proliferation and inhibited apoptosis;
\end{abstract}

Correspondence to: Professor Yan-Fu Wang, Department of Geriatrics, The First Affiliated Hospital of Dalian Medical University, 193 Lianhe Road, Dalian, Liaoning 116000, P.R. China E-mail: wangyanfu2000@163.com

Professor Ming-Yang Gao, Department of Dermatology, The First Affiliated Hospital of Dalian Medical University, 5 Longbin Road, Dalian, Liaoning 116000, P.R. China

E-mail: dlgaomingyang@126.com

*Contributed equally

Key words: EGF, MEK signaling pathway, RFPL3, hTERT, non-small cell lung cancer
PD98059 decreased RFPL3 and hTERT protein expression; and RFPL 3 overexpression increased the expression of hTERT and related MEK-pathway proteins. EGF upregulated RFPL3 and hTERT protein expression in NSCLC cells via the MEK pathway, promoted cell proliferation and inhibited apoptosis. RFPL3 overexpression increased expression of hTERT and related MEK signaling proteins (Ras, Raf, ERK and p-ERK), which implies that RFPL3 is a potential therapeutic target for NSCLC.

\section{Introduction}

The World Health Organization has reported that $~ 1.6$ million people succumb to lung cancer annually, which accounts for $19.4 \%$ of the total cancer mortality (1). The 5-year survival rate ranges from $50 \%$ for stage IA to $2 \%$ for stage IV, and the average 5-year overall survival rate of lung adenocarcinoma is $\sim 15 \%(2,3)$. Surgery, radiotherapy, chemoradiotherapy, molecular-targeted therapy and immunotherapy have been successful in curing lung cancer. However, most patients with advanced lung cancer have lost the chance for surgery, and radiotherapy and chemotherapy are less efficacious, leading to a very poor 5-year survival rate $(<15 \%)$. Non-small cell lung cancer (NSCLC) accounts for $\sim 87 \%$ of lung cancer; $\sim 60 \%$ of NSCLC diagnoses are discovered at advanced stages (4), with poor prognosis. Therefore, deep investigation of NSCLC pathogenesis and identification of new therapeutic targets is urgently needed.

Telomeres are protective structures at the ends of chromosomes, which consist of random repeat DNA sequences (TTAGGG) and associated proteins. The telomeres maintain chromosome length and stability, and defend chromosome ends from deleterious degradation and recombination or fusion. Telomerase, a key component in the forming of telomeres, can extend telomere DNA strands and repair broken ends of chromosomes, thereby extending, and even immortalizing, the lifespan of cells. Human telomerase reverse transcriptase (hTERT) mediates telomerase activation. Telomerase activity is very low or at almost undetectable levels in most normal cells, but may be overexpressed in tumor cells, which proliferate unlimitedly.

Many factors are directly or indirectly involved in the regulation of tumor growth and differentiation. Growth factors, 
especially the epidermal growth factor (EGF) family, growth factor receptor, oncogenes and anti-oncogenes are likely to affect cancer cell proliferation. EGF consists of 53 amino acid residues, coded on human chromosome 4 (4q25-27), and can be detected in most normal cells, human cancer tissue, blood, urine, and saliva (5). EGF can promote cell proliferation in tumors, and has an especially morphogenic effect on proliferation and clonality of NSCLC cells (6). Epidermal growth factor receptor (EGFR) is a member of the ErbB family of tyrosine kinase receptors, and is highly expressed in $27-83 \%$ of NSCLC, including $83 \%$ of squamous cell carcinoma, $\sim 40-50 \%$ of adenocarcinoma and large-cell lung carcinoma, but not in small cell lung carcinoma $(7,8)$. Reportedly, EGF increases hTERT protein expression in NSCLC cells, and can be blocked by EGFR tyrosine kinase inhibitors (TKIs) (9).

Ret finger protein-like 3 (RFPL3) is a hTERT-induced protein that helps regulate $h T E R T$ promoter activity and hTERT expression, to enhance telomerase activity and induce cancer cell proliferation (10). In the present study, we investigated whether EGF could upregulate RFPL3 and hTERT expression in human lung adenocarcinoma A549 and H1299 cells, and identified the involvement of the MEK signaling pathway in this process. We also investigated the relationship between RFPL3 overexpression and related MEK signaling proteins.

\section{Materials and methods}

Cell culture. A549 human lung adenocarcinoma cells and H1299 human NSCLC cells were kindly provided by the Regenerative Medicine Center, First Affiliated Hospital of Dalian Medical University. The cells were cultured in RPMI-1640 medium (Gibco; Thermo Fisher Scientific, Inc., Waltham, MA USA) supplemented with $10 \%$ fetal bovine serum (Gibco; Thermo Fisher Scientific, Inc.), $100 \mathrm{U} / \mathrm{ml}$ penicillin and $100 \mathrm{mg} / \mathrm{ml}$ streptomycin at $37^{\circ} \mathrm{C}$ in a $5 \% \mathrm{CO}_{2}$ humidified atmosphere. The cells were plated in 6-well plates for the activation of EGF (PeproTech, Inc., Rocky Hill, NJ, USA) and transfection studies, and were plated in 96-well plates for the MTT assay.

Cell treatment. We added EGF to the cells to final concentrations of 0 (control), $2.5,5,10,20 \mathrm{or} 50 \mathrm{ng} / \mathrm{ml}$ for $24 \mathrm{~h}$ or $48 \mathrm{~h}$. To inhibit EGFR, we added $10 \mu \mathrm{M}$ AG1478 (Selleck Chemicals, Shanghai, China) or $20 \mu \mathrm{M}$ erlotinib (Selleck Chemicals) $4 \mathrm{~h}$ prior to EGF treatment. To inhibit the MEK signaling pathway, we added $50 \mu \mathrm{M}$ PD98059 (Selleck Chemicals) $2 \mathrm{~h}$ prior to EGF treatment.

Western blot analysis. Cells were trypsinized and cell lysates were harvested in RIPA-SDS buffer supplemented with protease inhibitors and phosphatase inhibitors (Beijing Solarbio Science and Technology Co., Ltd., Beijing, China). The lysates were centrifuged at $12,000 \mathrm{x}$ for $20 \mathrm{~min}$, and the supernatants were then collected. Proteins were quantified using the BCA kit (Beijing Solarbio Science and Technology Co., Ltd.) according to the manufacturer's instructions. An equivalent amount of protein extract from each sample was electrophoresed by $12 \%$ SDS-PAGE and transferred to polyvinylidene difluoride membranes (Millipore, Billerica, MA, USA). The membranes were then blocked with 5\% non-fat dried milk in PBS/0.1\% Tween-20 for $1 \mathrm{~h}$, and incubated overnight at $4^{\circ} \mathrm{C}$ with the anti-RFPL3 (1:500; rabbit polyclonal; cat. no. 13215-1-AP; ProteinTech, Group, Inc., Chicago, IL, USA), anti-hTERT (1:1,000; cat. no. ARG54933; rabbit polyclonal; Arigo, Shanghai, China), anti-pan-Ras (1:20,000; mouse monoclonal; cat. no. 60309-1-lg; ProteinTech Group), anti-Raf1 (1:500; rabbit polyclonal; cat. no. 51140-1-AP; ProteinTech Group), anti-ERK1/2 (1:10,000; rabbit monoclonal; cat. no. ab184699; Abcam, Cambridge, MA, USA), anti-phospho-ERK1/2 (1:500; rabbit monoclonal; cat. no. ab32538; Abcam) or anti- $\beta$-actin $(1: 1,500$; rabbit monoclonal; cat. no. bs0061R; Bioss, Shanghai, China), respectively. Anti- $\beta$-actin was used as a loading control. The membranes were then washed three times with PBS/0.1\% Tween-20 (15 min each) and incubated with the corresponding secondary antibodies (horseradish peroxidase-conjugated, goat antibodies to rabbit and goat antibodies to mouse; 1:5,000; cat. nos. SA00001-2 and SA00001-1; ProteinTech Group) for $1 \mathrm{~h}$ at room temperature. After washing three times in PBS $/ 0.1 \%$ Tween-20, the membranes were then detected with ECL solution (Thermo Fisher Scientific). All the protein bands were densitometrically scanned and analyzed with ImageJ 1.44 software (National Institutes of Health, Bethesda, MD, USA).

RNA extraction and real-time qPCR assay. A549 and H1299 cells were treated with different final concentrations of EGF $(0,2.5,5,10,20$ or $50 \mathrm{ng} / \mathrm{ml})$ for $48 \mathrm{~h}$. Total RNA was extracted from these A549 and H1299 cells using RNAiso Plus (Takara Bio, Otsu, Japan) according to the manufacturer's protocol and was quantified with NanoDrop 2000 (Thermo Fisher Scientific). RNA ( $1 \mu \mathrm{g})$ was used as the template for cDNA synthesis; cDNA was reverse transcribed with the Primscript RT Reagent kit (Takara Bio). RT-qPCR reactions were performed on ABI StepOnePlus PCR instrument (Applied Biosystems; Thermo Fisher Scientific) for 40 cycles at $95^{\circ} \mathrm{C}$ for $5 \mathrm{sec}$, and at $60^{\circ} \mathrm{C}$ for $30 \mathrm{sec}$. Comparative quantification was determined using the $2^{-\Delta \Delta C T}$ method. Expression levels of RFPL3 and hTERT mRNA were standardized to GAPDH. Primer sequences are listed in Table I.

Cell viability and proliferation assay. For the MTT assay, A549 and H1299 cells were plated onto a 96-well plate at a cell density of 1,500 cells (A549) or 3,000 cells (H1299) per well with $0.2 \mathrm{ml}$ of supplemented RPMI-1640 per well. We treated the cells with different final concentrations of EGF $(0,2.5,5,10,20$ or $50 \mathrm{ng} / \mathrm{ml})$ for $48 \mathrm{~h}$, and then added $20 \mu \mathrm{l}$ of MTT $(5 \mathrm{mg} / \mathrm{ml}$; Sigma-Aldrich; Merck KGaA, Darmstadt, Germany) to each well and incubated them at $37^{\circ} \mathrm{C}$ for another $4 \mathrm{~h}$. The reaction was terminated by lysing the cells with $150 \mu \mathrm{l}$ of DMSO (Sigma-Aldrich; Merck KGaA), with gentle shaking for $10 \mathrm{~min}$. Optical density was measured at $490 \mathrm{~nm}$ in the spectrometer (BioTek Instruments, Inc. USA) to determine the absorbance of each well. Each separate treatment included five wells and was replicated three times on the 96-well plate.

Apoptosis analysis via flow cytometry. After treatment with different final concentrations of $\operatorname{EGF}(0,2.5,5,10,20$ or $50 \mathrm{ng} / \mathrm{ml}$ ) for $48 \mathrm{~h}$ in 6-well plates, A549 cells were trypsinized and harvested in cold PBS, collected by centrifugation 
Table I. Sequences of all primers used in this study.

Target gene

\section{GAPDH}

RFPL3

hTERT
Forward primer (5'-3')

Reverse primer (5'-3')

GCACCGTCAAGGCTGAGAAC
GCCCAATCGGCAGCTAGAGA
CATCGCCAGCATCATCAAAC

TGGTGAAGACGCCAGTGGA CTGTGTCGGCATCCAAGGTC GCCACGAACTGTCGCATGTA

GAPDH, glyceraldehyde-3-phosphate dehydrogenase; RFPL3, Ret finger protein like 3; hTERT, human telomerase reverse transcriptase.

A

EGF $(\mathrm{ng} / \mathrm{ml})$ A549 $\mid \begin{gathered}\text { RFPL3 (35 kDa) } \\ \text { hTERT (127 kDa) } \\ \beta \text {-actin (42 kDa) }\end{gathered}$
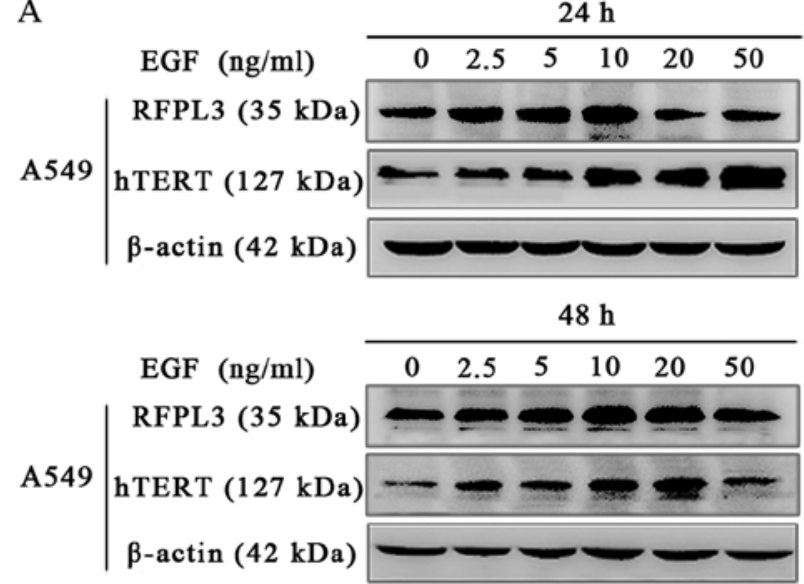

C
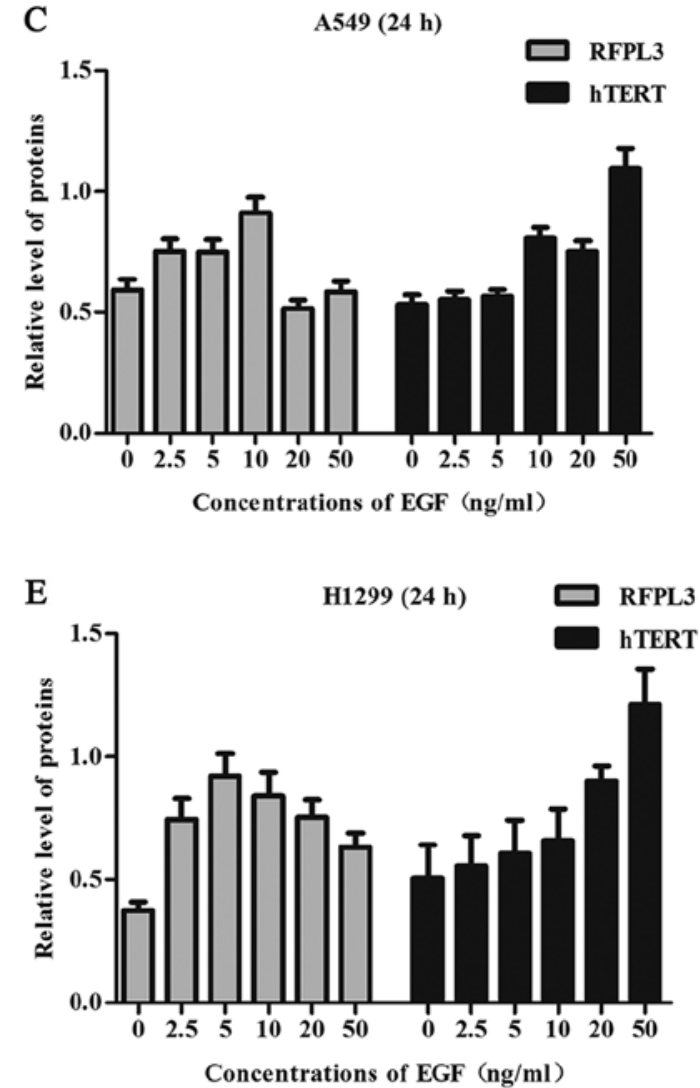

B

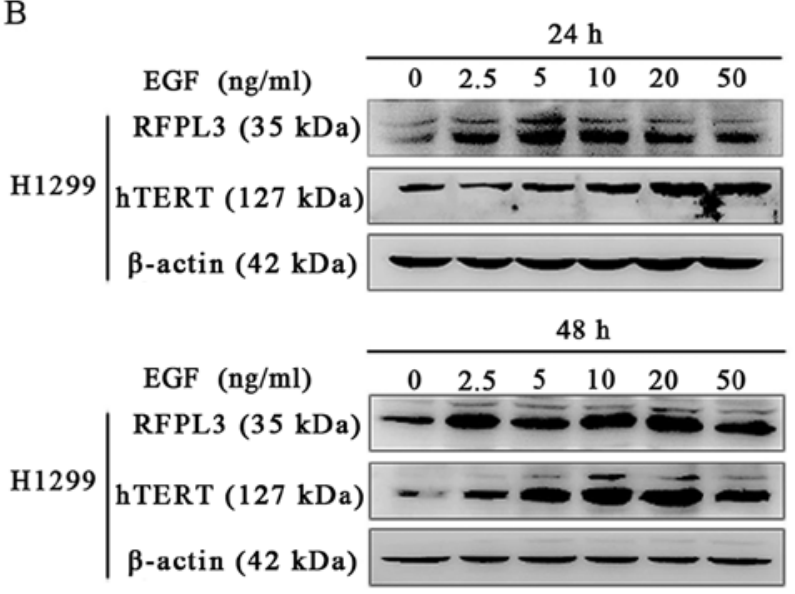

D

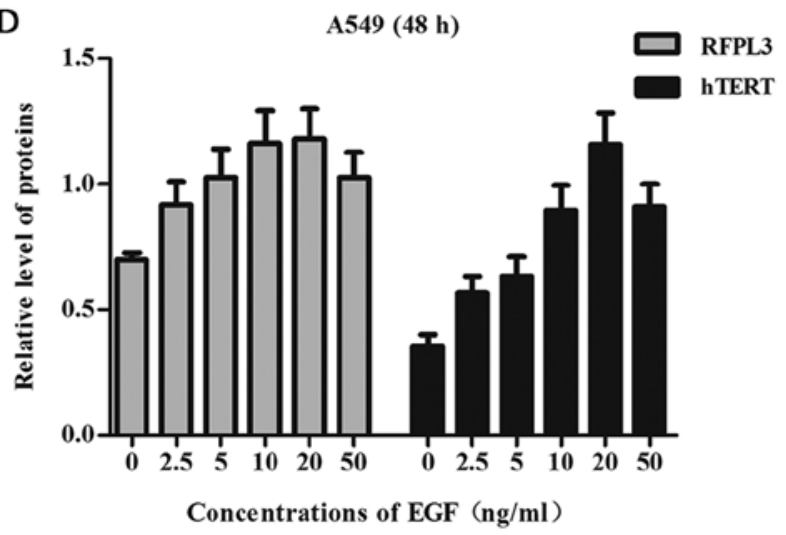

F

H1299 (48 h)

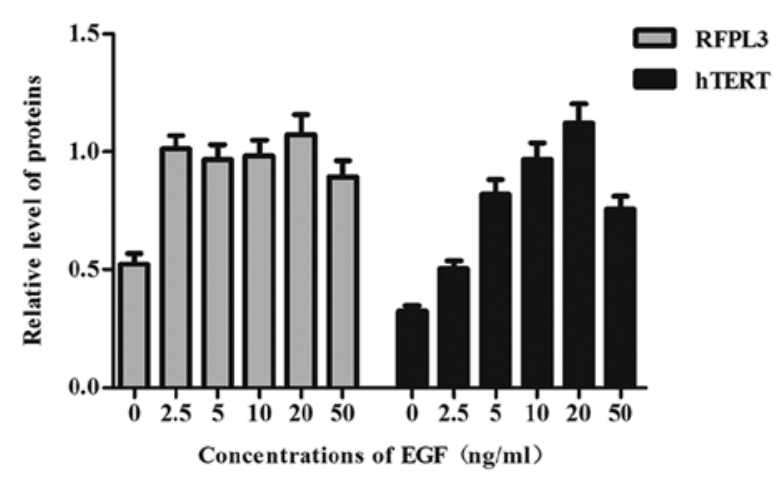

Figure 1. Western blot analysis of RFPL3 and hTERT expression in A549 and H1299 cells stimulated with different concentrations of EGF for $24 \mathrm{~h}$ or $48 \mathrm{~h}$. (A) A549 and (B) H1299 cells were respectively treated with different concentrations of EGF (0, 2.5, 5, 10, 20 or $50 \mathrm{ng} / \mathrm{ml}$ ) for $24 \mathrm{~h}$ or $48 \mathrm{~h}$. (C-F) Expression of RFPL3 and hTERT proteins were detected by western blot analysis. Results show that EGF stimulation significantly increased RFPL3 protein expression in the A549 and H1299 cells, with corresponding changes in hTERT expression. At 48 h, RFPL3 and hTERT proteins were expressed incrementally in a dose-dependent manner; $20 \mathrm{ng} / \mathrm{ml}$ was the optimal concentration. EGF, epidermal growth factor; RFPL3, Ret finger protein like 3; hTERT, human telomerase reverse transcriptase. 
A
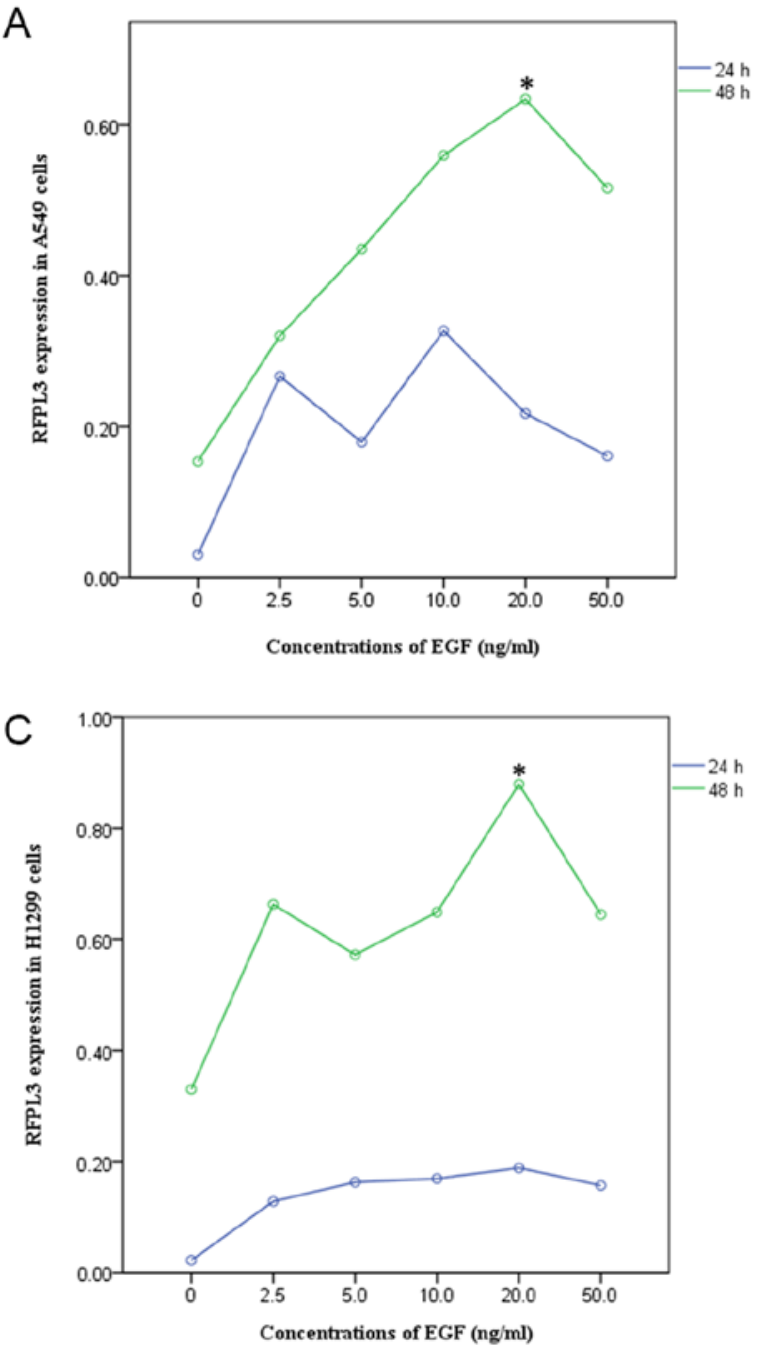
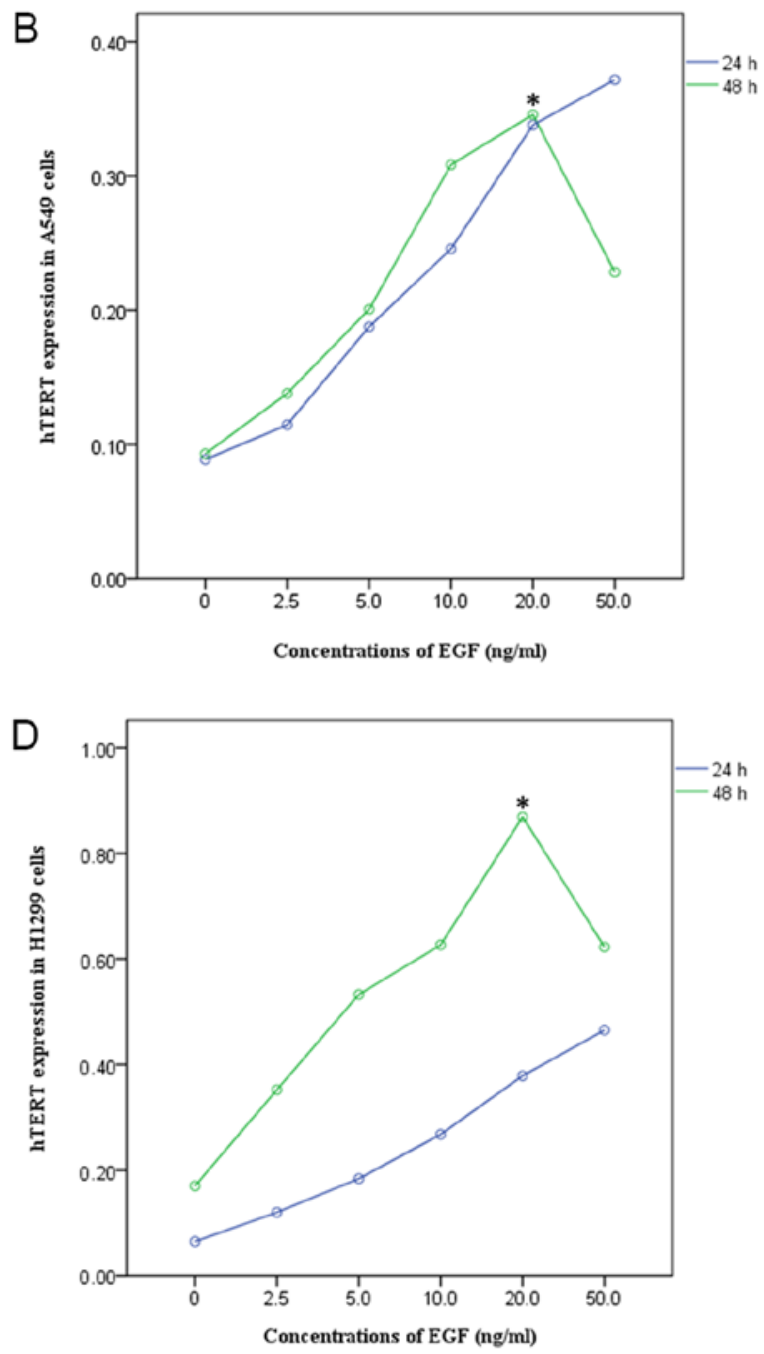

Figure 2. The expression of RFPL3 and hTERT proteins in A549 and H1299 cells when treated with different concentrations of EGF for $24 \mathrm{~h}$ or $48 \mathrm{~h}$. (A-D) EGF stimulation significantly increased RFPL3 protein expression in A549 and H1299 cells; expression of hTERT exhibited a corresponding tendency. When stimulated for $48 \mathrm{~h}$, RFPL3 and hTERT proteins were expressed incrementally in a dose-dependent manner, with $20 \mathrm{ng} / \mathrm{ml}$ as the optimal concentration. Data are expressed as the mean \pm standard deviation of three independent experiments using the factorial design statistical method. " $\mathrm{P}<0.05$.
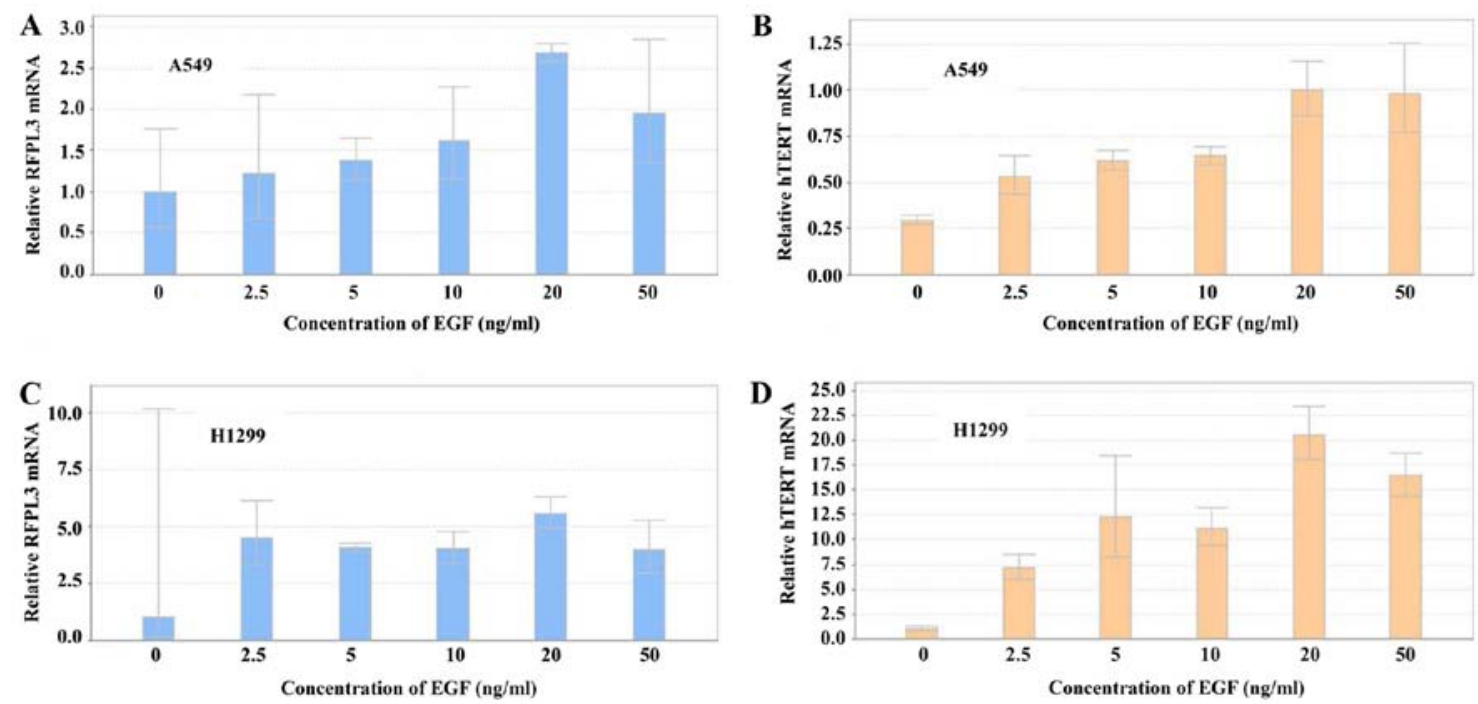

Figure 3. mRNA levels of RFPL3 and hTERT in A549 and H1299 cells as detected by RT-qPCR. (A-D) A549 and H1299 cells were treated with different concentrations of EGF $(0,2.5,5,10,20$ or $50 \mathrm{ng} / \mathrm{ml})$ for $48 \mathrm{~h}$. Expression of $R F P L 3$ and $h T E R T$ mRNA was determined by RT-qPCR. Results showed that EGF markedly increased RFPL3 and $h T E R T$ mRNA expression, consistent with the increased protein expression. EGF, epidermal growth factor; RFPL3, Ret finger protein like $3 ; h T E R T$, human telomerase reverse transcriptase. 
A

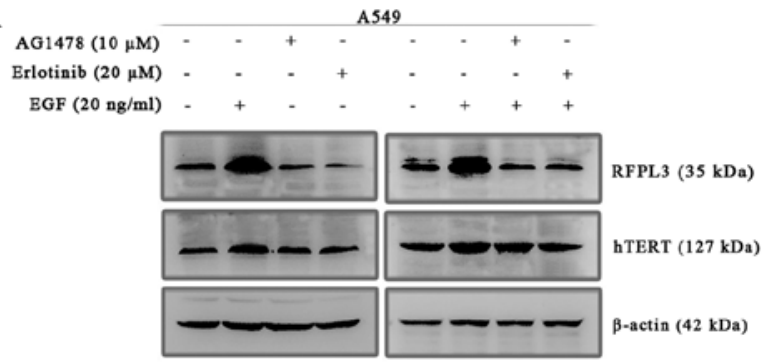

C

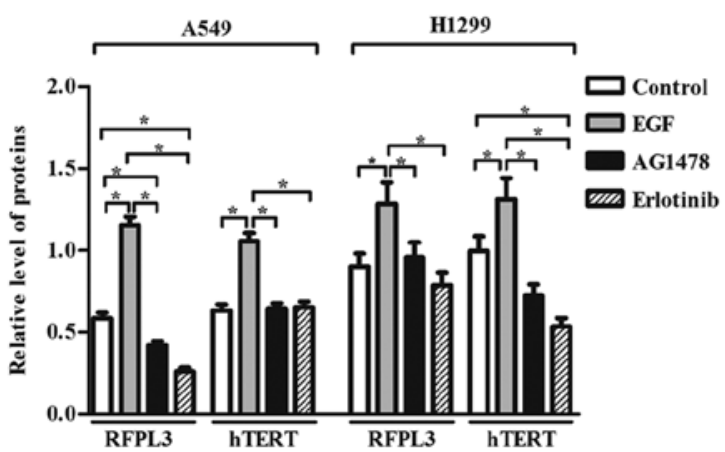

B

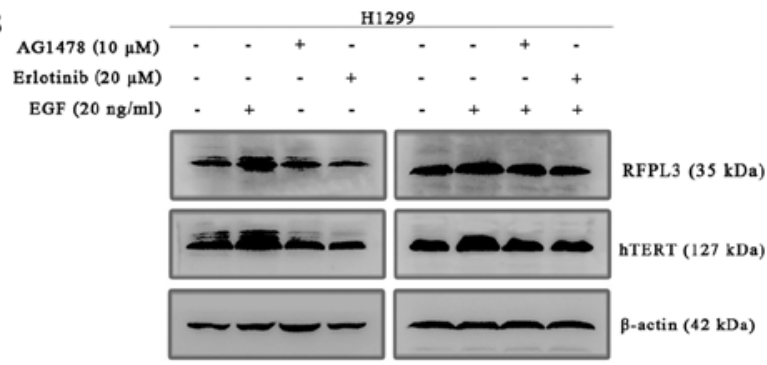

D
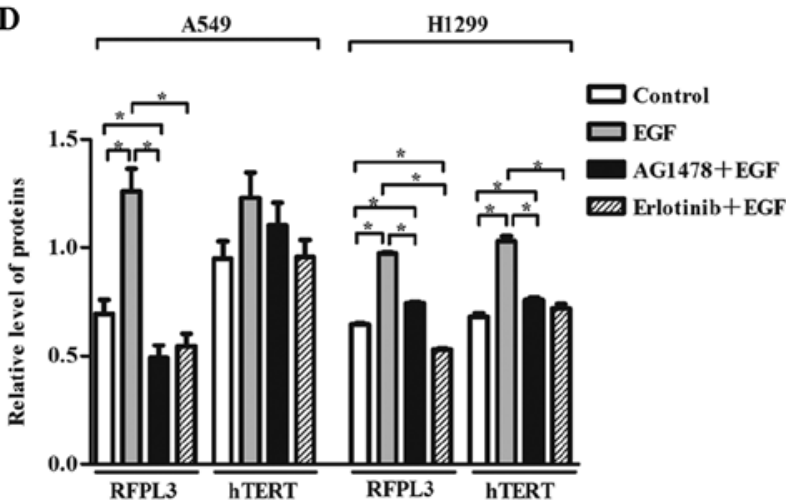

Figure 4. Western blot analysis of RFPL3 and hTERT expression in A549 and H1299 cells with or without EGFR inhibitors. (A and B) A549 and H1299 cells were incubated with or without AG1478 $(10 \mu \mathrm{M})$ and erlotinib $(20 \mu \mathrm{M})$ for 4 hs prior to a 48 -h incubation with EGF (20 ng/ml). EGFR kinase inhibitors partially inhibited expression of RFPL3 and hTERT proteins, which was reversible by EGF. (C and D) Histograms indicate the relative quantitative expression of RFPL3 and hTERT proteins. Data are expressed as the mean \pm standard deviation of three independent experiments. "P<0.05. EGF, epidermal growth factor; RFPL3, Ret finger protein like 3; hTERT, human telomerase reverse transcriptase.

(5 min at $800 \mathrm{x} \mathrm{g}$ ), resuspended at $1 \times 10^{6}$ cells $/ \mathrm{ml}$ in $100 \mu 11 \mathrm{X}$ binding buffer, and stained with $5 \mu \mathrm{l}$ of Annexin V-FITC for $10 \mathrm{~min}$ in room temperature, protected from light. We then incubated them with $5 \mu \mathrm{l}$ of PI solution for $5 \mathrm{~min}$, added PBS to a volume of $500 \mu \mathrm{l}$ and immediately analyzed the cells using a flow cytometer.

Transfection. The GFP-marked RFPL3-overexpression plasmid and its control vector were designed and produced by Genepharma Corporation, Suzhou, China. A549 and H1299 cells were plated in 6 -well plates at $1.2 \times 10^{5}$ cells/well in $0.2 \mathrm{ml}$ of medium overnight. Transfection was performed when cells achieved $80-90 \%$ confluency. Cells were assigned into blank control, negative control and transfected groups. The negative control was treated with control vector. Cells were transfected with RFPL3-overexpression plasmids or control vector, with serum-free medium, using the Invitrogen ${ }^{\mathrm{TM}}$ Lipofectamine 2000 system (Thermo Fisher Scientific, Inc.) according to the manufacturer's instructions. The medium was replaced with complete medium $6 \mathrm{~h}$ later.

Statistical analysis. The experiments were repeated at least three times. Statistical analyses were performed using the SPSS software (version 22.0) (IBM Corp., Armonk, NY, USA), and values from three independent experiments at least are represented as the mean \pm standard deviation. Unpaired Student's t-test and one-way analysis of variance (ANOVA) was performed respectively for the two groups and three or more than three groups to evaluate the statistical significance. A value of $\mathrm{P}<0.05$ was considered to indicate statistical significance.

\section{Results}

EGF upregulates expression of RFPL3 and hTERT proteins in NSCLC cells. A549 and H1299 cells were cultured in the presence of EGF concentrations ranging from 0 to $50 \mathrm{ng} / \mathrm{ml}$ for $24 \mathrm{~h}$ or $48 \mathrm{~h}$. EGF stimulation significantly increased RFPL3 protein expression; expression of hTERT exhibited a corresponding tendency (Figs. 1 and 2). This suggests a positive correlation between expressions of RFPL3 and hTERT. Intriguingly, the protein levels increased when stimulated for $24 \mathrm{~h}$, but the activity appeared to be unstable. When stimulated for $48 \mathrm{~h}$, RFPL3 and hTERT proteins were expressed incrementally in a dose-dependent manner, with $20 \mathrm{ng} / \mathrm{ml}$ as the optimal concentration.

Expression of RFPL3 and hTERT mRNA was analyzed by RT-qPCR in A549 and H1299 cells after treatment with different concentrations of EGF for $48 \mathrm{~h}$ (Fig. 3). EGF induced a marked increase in RFPL3 and hTERT mRNA expression, which was in accord with their protein levels. The highest mRNA expression came at $20 \mathrm{ng} / \mathrm{ml}$ of EGF for $48 \mathrm{~h}$.

EGF-induced upregulation of RFPL3 and hTERT is attenuated by pretreatment with EGFR inhibitors, AG1478 and erlotinib. To clarify the effect of EGF on RFPL3 and hTERT expression, we incubated A549 and H1299 cells with or without AG1478, and with or without erlotinib, in the presence of EGF. EGF (20 ng/ml) markedly upregulated RFPL3 and hTERT (Fig. 4). When treated with EGFR-TKIs [AG1478 $(10 \mu \mathrm{M})$ or erlotinib $(20 \mu \mathrm{M})$ ] alone, RFPL3 and hTERT expression was attenuated. When treated with a combination of EGFR-TKIs and EGF, EGF-induced upregulation 

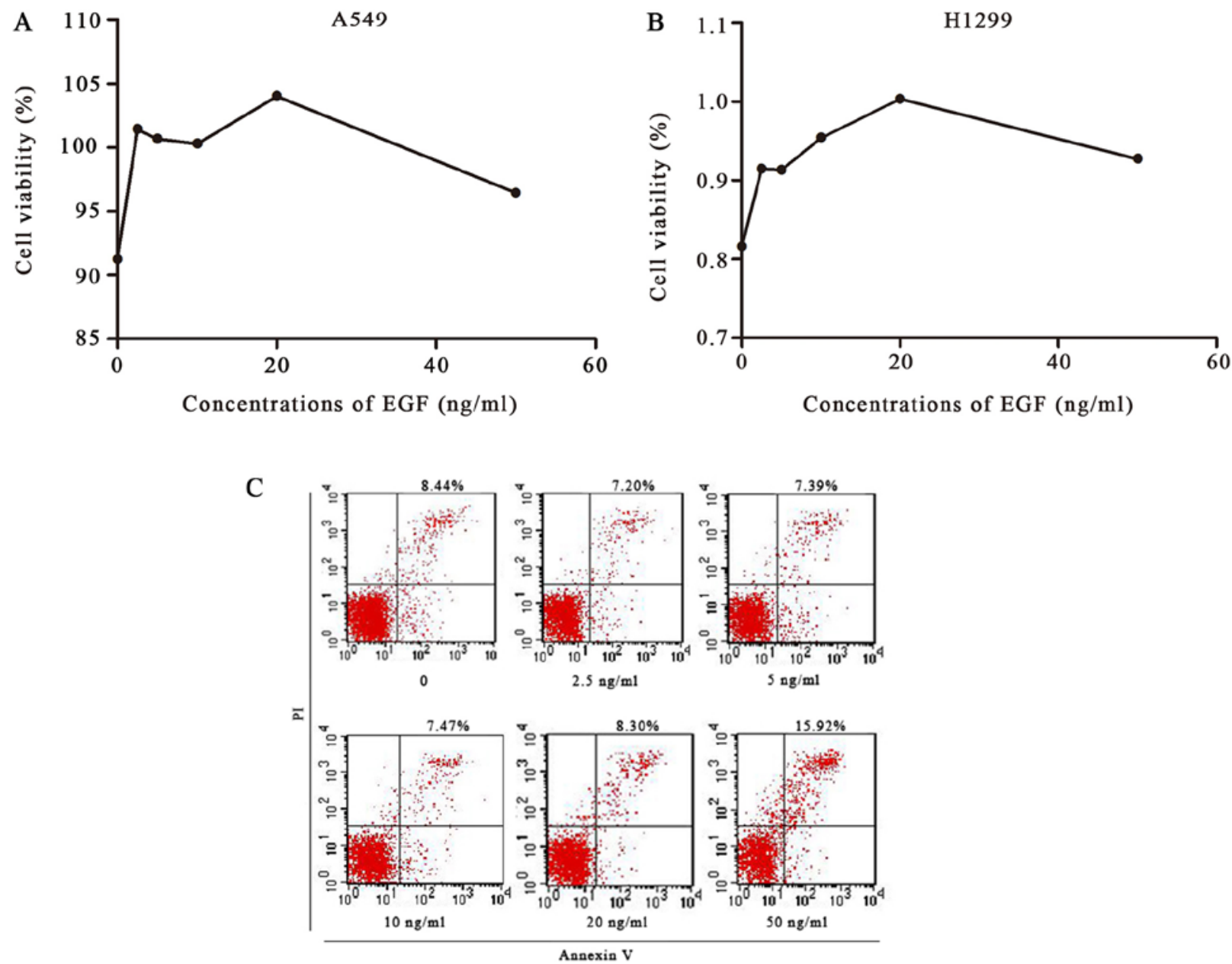

Figure 5. Cell viability was detected by MTT assay, and apoptosis of A549 cells was detected by flow cytometry. (A and B) A549 and H1299 cells were treated with $0,2.5,5,10,20$ or $50 \mathrm{ng} / \mathrm{ml}$ of EGF for $48 \mathrm{~h}$. An MTT assay showed that EGF significantly enhanced the proliferation of A549 and H1299 cells, with a maximum increase at $20 \mathrm{ng} / \mathrm{ml}$ EGF. (C) A549 cells were treated with 0,2.5, 5, 10, $20 \mathrm{or} 50 \mathrm{ng} / \mathrm{ml}$ of EGF for $48 \mathrm{~h}$; apoptosis was then detected by flow cytometry. Compared with the control group, 2.5, 5, 10 and $20 \mathrm{ng} / \mathrm{ml}$ of EFG inhibited apoptosis, whereas $50 \mathrm{ng} / \mathrm{ml}$ of EGF increased apoptosis. EGF, epidermal growth factor.

of RFPL3 and hTERT was also attenuated, but was slightly higher than the levels in cells treated with AG1478 or erlotinib alone. These findings suggest that EGFR-TKIs partially and reversibly inhibit RFPL3 and hTERT expression.

EGF significantly promotes cell proliferation and affects apoptosis in NSCLC. To investigate the effect of EGF on cell growth, we detected the viability of A549 and H1299 cells using the MTT assay. EGF significantly enhanced cell proliferation, with maximum increased growth at $20 \mathrm{ng} / \mathrm{ml}$ EGF, which was consistent with the other results in this study (Fig. 5). Compared with the control group, EGF doses of 2.5, 5, 10 and $20 \mathrm{ng} / \mathrm{ml}$ inhibited apoptosis, whereas $50 \mathrm{ng} / \mathrm{ml}$ increased apoptosis.

EGF upregulates expression of RFPL3 and hTERT via the MEK signaling pathway. To determine whether EGF-induced upregulation of RFPL3 and hTERT proteins in NSCLC cells was related to the MEK signaling pathway, we pretreated A549 and H1299 cells with an MEK-specific inhibitor [PD98059 $(50 \mu \mathrm{M})]$. Expression of RFPL3 and hTERT was significantly increased by EGF treatment (Fig. 6). The treatment of A549 and H1299 cells with PD98059 decreased expression of p-ERK protein, and also markedly decreased EGF-induced upregulation of RFPL3 and hTERT, without affecting total ERK protein.

Effects of RFPL3 overexpression on MEK signaling proteins. To further explore the effects of RFPL3 overexpression in NSCLC cells on related proteins in the MEK pathway, we transfected A549 and H1299 cells with a RFPL3-overexpression plasmid, and then detected the transfection efficiency by fluorescence microscope and examined the expression of RFPL3, hTERT, Ras, Raf, ERK, and p-ERK by western blot analysis. After a successful transfection ( $>80 \%$ of cells transfected; Fig. 7), RFPL3 protein was overexpressed, which promoted hTERT expression and also increased expression of the related MEK pathway proteins (Fig. 8). These results imply that control for expression of RFPL3 and hTERT, and for these MEK signaling proteins, may be inter-related.

\section{Discussion}

EGFR is an important therapeutic target in lung and other cancers. Therapies targeted to EGFR, including 

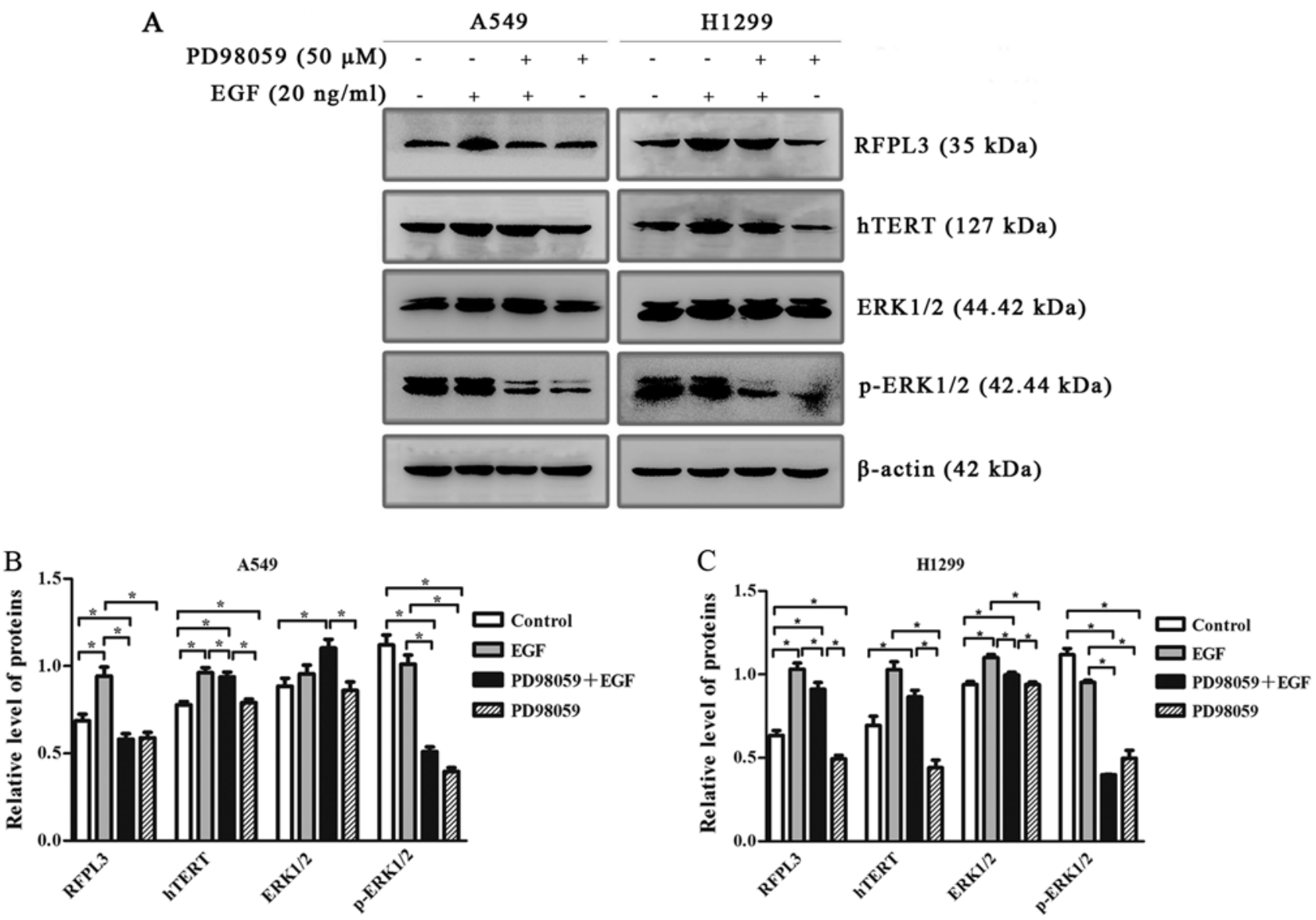

Figure 6. Western blot analysis of RFPL3, hTERT, ERK and p-ERK protein expression in A549 and H1299 cells, treated with or without MEK-specific inhibitor (PD98059). (A) A549 and H1299 cells were treated with PD98059 (50 $\mu \mathrm{M})$ for $2 \mathrm{~h}$, and then with $20 \mathrm{ng} / \mathrm{ml}$ EGF for $48 \mathrm{~h}$; expression levels of RFPL3, hTERT, ERK and p-ERK proteins were then detected by western blot analysis. PD98059 decreased expression of p-ERK without affecting total ERK protein, and also markedly decreased EGF-induced upregulation of RFPL3 and hTERT in H1299 cells, but did not significantly decrease hTERT expression. (B and C) Histograms indicate relative quantitative expression of these proteins. Data are expressed as the mean \pm standard deviation of three independent experiments. ${ }^{*} \mathrm{P}<0.05$ EGF, epidermal growth factor; RFPL3, Ret finger protein like 3; hTERT, human telomerase reverse transcriptase.

A
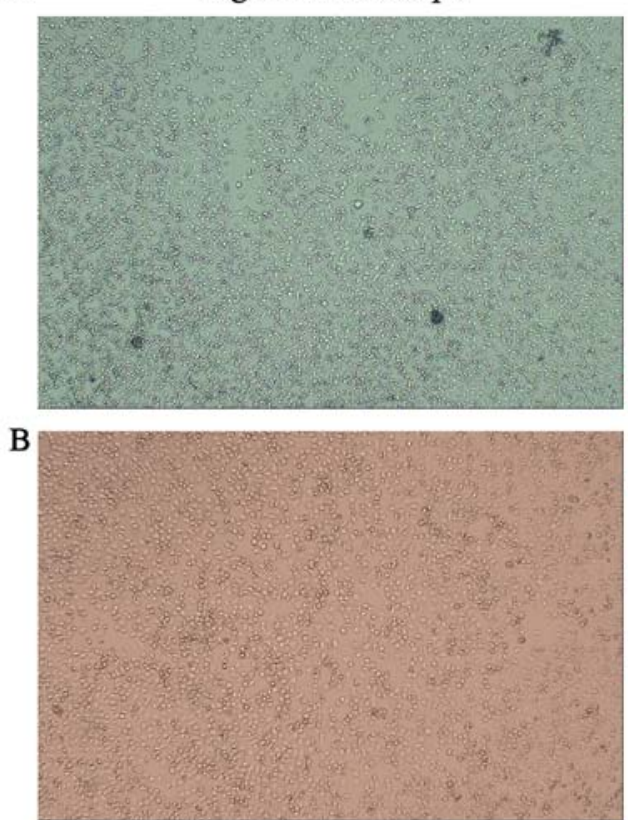

Light microscope

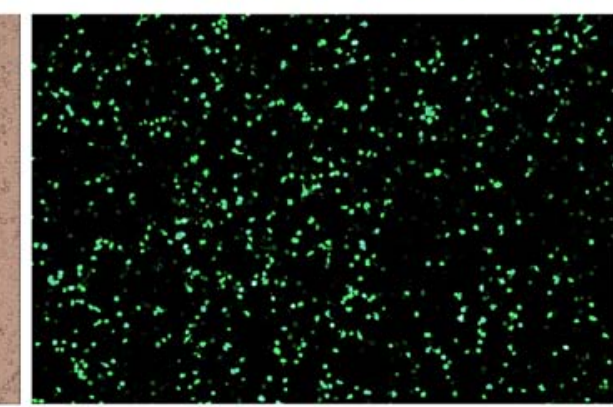

Fluorescence microscope

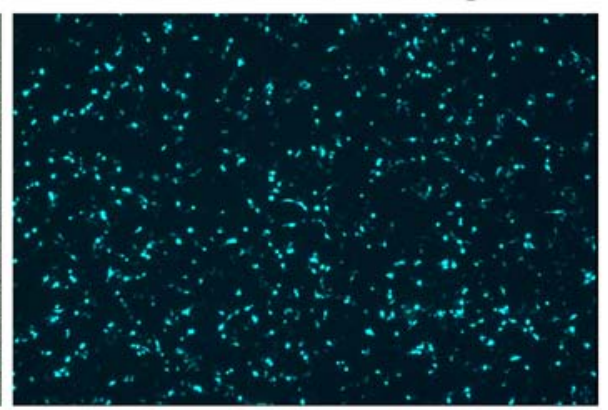

A549 $(\times 100)$

H1299 (×100)

Figure 7. We transiently transfected a RFPL3 (GFP) plasmid into (A) A549 and (B) H1299 cells, and then detected the transfection efficiency by fluorescence microscope. The representative images showed that the transfection efficiency of A549 and H1299 cells was above 80\%, which indicates successful transfection. RFPL3, Ret finger protein like 3 . 
A
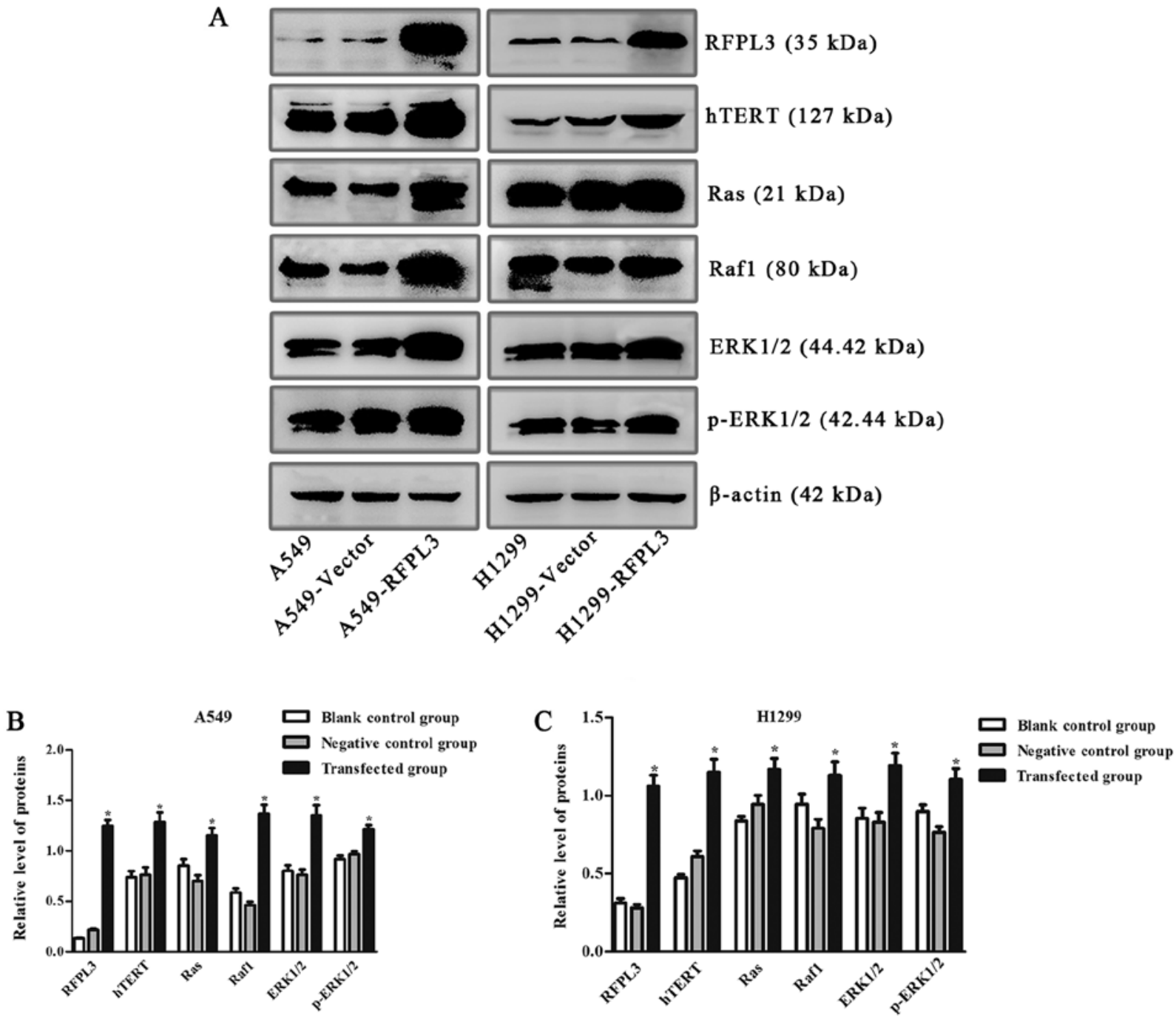

Figure 8. Expression of hTERT and related proteins in the MEK signaling pathway in A549 and H1299 cells transfected with an RFPL3-overexpression plasmid. (A) Western blot analysis was used to detect expression of RFPL3, hTERT, Ras, Raf, ERK and p-ERK. Compared with the blank control and negative control groups, RFPL3 in transfected cells was overexpressed, and expression of hTERT, Ras, Raf, ERK and p-ERK also increased. (B and C) Histograms indicate relative quantitative expression of these proteins. Data are expressed as the mean \pm standard deviation of three independent experiments. "P<0.05, compared with the negative control group. RFPL3, Ret finger protein like 3; hTERT, human telomerase reverse transcriptase.

low-molecular-weight TKIs and monoclonal antibodies, have been successfully implemented in the clinic for more than a decade. First-generation TKIs include gefinitib, erlotinib and icotinib; second-generation TKIs include afatinib and dacomitinib.

NSCLC tissues with EGFR mutations are more sensitive to EGFR-TKIs. Reportedly, the NSCLC response rate to EGFR-TKIs is $70-80 \%$, but only $10-20 \%$ for NSCLC tumors with wild-type EGFR (11), which restricts the use of this treatment. In addition, EGFR-TKI resistance, including both primary and acquired resistance, is increasingly serious. Primary resistance may be caused by oncogenic $K$-ras mutation, $M E T$ amplification, BIM gene expression and polymorphism, and PTEN absence. EGFR T790M secondary mutations may cause acquired resistance. RAF/MEK/ERK signaling pathway inhibitors, such as respective inhibitors of Raf, MEK, and ERK, have been investigated in clinical trials; among these, MEK inhibitors have the strongest cytotoxic effects, but cancer cells with $K$-ras mutations are less sensitive to MEK inhibitors. Our present results showed that, when treated with a MEK-specific inhibitor (PD98059), hTERT expression in A549 cells did not decrease significantly.

Binding by EGF changes the intramolecular conformation of EGFR, which activates intracellular tyrosine kinase, and sequentially triggers several signaling pathways, including RAS-MAPK, PI3K-Akt, PLC $\gamma$ and JAKs-STATs. Thus, EGF regulates transcription factors and activates gene transcription or related protein expression by adapter proteins and enzyme cascade reactions, ultimately regulating cell proliferation, differentiation, apoptosis, and angiogenesis (12-14). The activation of the Ras/MAPK (MEK) signaling transduction pathway is associated with cell proliferation. The MEK signaling pathway mainly regulates cell proliferation and differentiation through phosphorylation and dephosphorylation. The underlying mechanisms of the MEK pathway in promoting tumorigenesis include increasing tumor cell proliferation, inhibiting apoptosis, promoting angiogenesis and inducing tissue invasion (15).

Human lung adenocarcinoma A549 cells contain wild-type EGFR and mutant-type $K$-ras, whereas $\mathrm{H} 1299$ cells have no 
detected mutation. Mutation of oncogene $K$-ras is the major driver of growth and proliferation in lung cancer cells. About $25 \%$ of patients with NSCLC have $K$-ras mutations which are associated with poor prognoses (16). The oncogene $K$-ras is associated with multiple signaling pathways, such as Raf/MEK/ERK, PI3K/AKT/mTOR, and RALGDS/RAL, that affect tumor development and progression.

Telomerase contains three important components: Human telomerase RNA (hTR), telomerase TP1 and human telomerase reverse transcriptase (hTERT). hTERT is the key component that determines telomerase activity. Although expression of hTERT is low or non-existent in normal cells, it is overexpressed in tumor cells, which suggests that hTERT activation is a key step in the somatic cell transformation to tumor cells. hTERT is the catalytic subunit of telomerase responsible for telomerase activation, telomere elongation, or even cell immortality. Activation of telomerase due to activation of hTERT would be a pivotal step in tumor occurrence and development. An in vitro study $(17,18)$ has indicated that drug resistance in lung cancer may be directly related to activation of telomerase. Ectopic overexpression of TERT in cancer cell lines makes them insensitive to radiotherapy and chemotherapy drugs.

The RFPL3 gene (located at human 22q12.3) has not been widely studied. RFPL3 belongs to the RFPL protein family (RFPL1, RFPL2 and RFPL3), and is homologous to $\mathrm{RFP}$, which is a nucleoprotein that helps regulate cell proliferation, differentiation and tumorigenesis. RFPL1, RFPL2 and RFPL3 present high transcriptional levels in the human cerebral cortex, where the highest concentration is in the developing cerebrum frontal lobe, due to the effect of transcription factor Pax6 (19). RFPL3 is reported to clearly enhance integration activity of the human immunodeficiency virus pre-integration complex protein in infected cells in vitro, which implies that RFPL3 overexpression would reinforce HIV infectivity (20). RFPL3 protein has been recently associated with occurrence and development of lung cancer. RFPL3 can regulate expression of the hTERT gene in NSCLC cells and drive hTERT promoter transcription, thus increasing telomerase activity and length, and promoting proliferation of tumor cells.

In the present study, we found that EGF stimulation significantly increased the levels of RFPL3 and hTERT proteins in NSCLC cells, and increased cell proliferation while inhibiting apoptosis; but EGFR TKIs could block its function.

Furthermore, we present novel evidence that EGF activates MEK signaling pathway; that EGF upregulates expression of RFPL 3 and hTERT proteins through the activation of MEK signaling pathway; and that overexpression of RFPL3 protein increases expression of hTERT and related proteins in MEK pathway.

Our results suggest that stimulation of A549 and H1299 cells with EGF at $24 \mathrm{~h}$ increased RFPL3 and hTERT expression but the activity appeared to be unstable-probably due to the lack of enough affinity. At 48 h, expression of RFPL3 and hTERT increased progressively, and was time- and concentration-dependent within concentrations of 0-20 ng/ml EGF. When treated with EGF at $50 \mathrm{ng} / \mathrm{ml}$, production of RFPL3 and hTERT proteins declined, cell viability decreased and apoptosis increased. Accordingly, we conjectured that constant EGF stimulation decreases cell sensitivity to EGFR.
We also found that EGFR TKIs (AG1478, erlotinib) partially and reversibly inhibited expression of RFPL3 and hTERT proteins, but did not completely stop their expression, which may reflect decreased sensitivity to EGFR TKIs and increased drug resistance.

A previous study showed that RFPL3 could drive hTERT promoter transcriptional activity, thus increasing its expression and contributing to development of NSCLC (10). Our results showed that RFPL3 overexpression increased hTERT expression; expression of RFPL3 and hTERT were positively correlated. The MEK signaling pathway is pivotal in this process. EGF binds with a specific cytomembrane receptor (EGFR), activates the downstream MEK signaling proteins (RAS/RAF/MEK/ERK), and then increases expression of ERK, and upregulates RFPL3 and hTERT proteins. Overexpressed RFPL3 protein also increased expression of upstream molecules (Ras, Raf) in the MEK signaling pathway, and sequentially increased ERK expression, which activates expression of growth-regulated oncoproteins.

In conclusion, our results indicate that EGF upregulates expression of RFPL 3 and hTERT proteins in NSCLC cells via the MEK signaling pathway, promotes cell proliferation and inhibits apoptosis. Our experiments demonstrated the link between EGFR and hTERT, indicating that EGF may upregulate hTERT expression and activity through RFPL3. Moreover, RFPL3 overexpression increases related MEK-signaling proteins (Ras, Raf, ERK, p-ERK), and promotes cell proliferation and differentiation. RFPL3 could thus be a therapeutic target in NSCLC, and have a role in NSCLC drug resistance.

\section{Acknowledgements}

We are very thankful to the Regenerative Medicine Center, First Affiliated Hospital of Dalian Medical University for providing us with two human NSCLC cell lines, A549 and H1299.

\section{Funding}

No funding was received.

\section{Availability of data and materials}

The datasets used during the present study are available from the corresponding author upon reasonable request.

\section{Authors' contributions}

CL, YQ, YFW and MYG conceived and designed the study. $\mathrm{CL}$ performed the experiments. CL and YQ wrote the manuscript. CL, YQ, HZ, YFW and MYG reviewed and edited the manuscript. All authors read and approved the manuscript and agree to be accountable for all aspects of the research in ensuring that the accuracy or integrity of any part of the work are appropriately investigated and resolved.

\section{Ethics approval and consent to participate}

This article contains no studies with human participants performed by any of the authors. 


\section{Consent for publication}

No human participants were involved in this study.

\section{Competing interests}

The authors declare that they have no competing interests.

\section{References}

1. World Health Organization, International Agency for Research on Cancer. Press Release No. 224, Lyon/London, 3 February 2014.

2. Minna JD and Schiller JH: Lung Cancer: Harrison's Principles of Internal Medicine. 17th edition. pp551-562, 2008.

3. Goldstraw P, Crowley J, Chansky K, Giroux DJ, Groome PA Rami-Porta R, Postmus PE, Rusch V, Sobin L and International Association for the Study of Lung Cancer International Staging Committee; Participating Institutions: The IASLC lung cancer staging project: Proposals for the revision of the TNM stage groupings in the forthcoming (seventh) edition of the TNM classification of malignant tumours. J Thorac Oncol 2: 706-714, 2007.

4. DeSantis CE, Lin CC, Mariotto AB, Siegel RL, Stein KD, Kramer JL, Alteri R, Robbins AS and Jemal A: Cancer treatment and survivorship statistics, 2014. CA Cancer J Clin 64: 252-271, 2014.

5. Hirata Y, Moore GW, Bertagna C and Orth DN: Plasma concentrations of immunoreactive human epidermal growth factor (urogastrone) in man. J Clin Endocrinol Metab 50: 440-444, 1980 .

6. Xiqing Li, Zunlan Zhao, Mingyue Liu, et al: Epidermal growth factor affects the growth and proliferation of non-small cell lung cancer A549 and H23 cells. Chinese Journal of applied diagnosis and treatment 29: 562-564, 2015 (In Chinese).

7. Bethune G, Bethune D, Ridgway N and Xu Z: Epidermal growth factor receptor (EGFR) in lung cancer: An overview and update. $\mathrm{J}$ Thorac Dis 2: 48-51, 2010.

8. Lee HJ, Xu X, Choe G, Chung DH, Seo JW, Lee JH, Lee CT, Jheon S, Sung SW and Chung JH: Protein overexpression and gene amplification of epidermal growth factor receptor in nonsmall cell lung carcinomas: Comparison of four commercially available antibodies by immunohistochemistry and fluorescence in situ hybridization study. Lung Cancer 68: 375-382, 2010.

9. Hsu CP, Lee LW, Tang SC, Hsin IL, Lin YW and Ko JL: Epidermal growth factor activates telomerase activity by direct binding of Ets-2 to hTERT promoter in lung cancer cells. Tumour Biol 36: 5389-5398, 2015.
10. Chen W, Lu J, Qin Y, Wang J, Tian Y, Shi D, Wang S, Xiao Y, Dai M, Liu L, et al: Ret finger protein-like 3 promotes tumor cell growth by activating telomerase reverse transcriptase expression in human lung cancer cells. Oncotarget 5: 11909-11923, 2014.

11. Mitsudomi T and Yatabe Y: Mutations of the epidermal growth factor receptor gene and related genes as determinants of epidermal growth factor receptor tyrosine kinase inhibitors sensitivity in lung cancer. Cancer Sci 98: 1817-1824, 2007.

12. Vivanco I and Sawyers CL: The phosphatidylinositol 3-Kinase AKT pathway in human cancer. Nat Rev Cancer 2: 489-501, 2002.

13. Murray SA, Yang S, Demicco E, Ying H, Sherr DH, Hafer LJ, Rogers AE, Sonenshein GE and Xiao ZX: Increased expression of MDM2, cyclin D1, and $\mathrm{p} 27^{\mathrm{Kip} 1}$ in carcinogen-induced rat mammary tumors. J Cell Biochem 95: 875-884, 2005.

14. Anderson D, Koch CA, Grey L, Ellis C, Moran MF and Pawson T: Binding of SH2 domains of phospholipase $\mathrm{C}$ gamma 1, GAP, and Src to activated growth factor receptors. Science 250: 979-982, 1990.

15. Yuqin Hao and Huixia Zheng: The mechanism research of MAPK/ERK signaling pathways in cancer treatment. China J Leprosy and Skin Diseases 7: 490-493, 2012 (In Chinese).

16. Roberts PJ and Stinchcombe TE: KRAS mutation: Should we test for it, and does it matter? J Clin Oncol 31: 1112-1121, 2013.

17. Loboda A, Nebozhyn M, Klinghoffer R, Frazier J, Chastain M, Arthur W, Roberts B, Zhang T, Chenard M, Haines B, et al: A gene expression signature of RAS pathway dependence predicts response to PI3K and RAS pathway inhibitors and expands the population of RAS pathway activated tumors. BMC Med Genomics 3: 26, 2010.

18. Matallanas D and Crespo P: New druggable targets in the Ras pathway? Curr Opin Mol Ther 12: 674-683, 2010.

19. Bonnefont J, Nikolaev SI, Perrier AL, Guo S, Cartier L, Sorce S, Laforge T, Aubry L, Khaitovich P, Peschanski M, et al: Evolutionary forces shape the human RFPL1,2,3 genes toward a role in neocortex development. Am J Hum Genet 83: 208-218, 2008.

20. Tan BH, Suzuki Y, Takahashi H, Ying PH, Takahashi C, Han Q Chin WX, Chao SH, Sawasaki T, Yamamoto N and Suzuki Y: Identification of RFPL3 protein as a novel E3 ubiquitin ligase modulating the integration activity of human immunodeficiency virus, type 1 preintegration complex using a microtiter plate-based assay. J Biol Chem 289: 26368-26382, 2014.

This work is licensed under a Creative Commons Attribution-NonCommercial-NoDerivatives 4.0 International (CC BY-NC-ND 4.0) License. 